\title{
Study of Magnetoacoustic Tomography with Injecting Current by Experiment
}

\author{
Xin Huang ${ }^{1,2}$ \\ ${ }^{1}$ Institute of Electrical Engineering, Chinese Academy \\ of Sciences; ${ }^{2}$ Graduate University of Chinese Academy \\ of Sciences \\ Beijing, China
}

\begin{abstract}
Magnetoacoustic Tomography (MAT) is a new imaging method. MAT combines the good contrast of electrical impedance tomography with the good spatial resolution of ultrasound imaging. Magnetoacoustic Tomography includes Magnetoacoustic Tomography with magnetic induction and Magnetoacoustic Tomography with injecting current. In this paper we researched the later imaging method mainly; in paper we studied the parameters of AC power supply, which was injected to the imaging sample. At the same time, the waveforms of experiment were given and analyzed, which provides theoretical basis for the selection of power supply used in the subsequent experiments.
\end{abstract}

Keywords-Lorentz force; Magnetoacoustic tomography; Pulse width; ultrasonic signal.)

\section{INTRODUCTION}

By researches we know that electrical impedance property of biological tissue can provide structure, functional and pathological information of the tissue. So by measuring changes of electrical impedance property of biological tissue, we can know whether there are lesions in the tissue. In recent years, the noninvasively image using electrical properties of biological tissues has become a hot area of research. In order to obtain high-quality images of electrical parameters, various new approaches have been explored, including the electrical impedance tomography (EIT), [1,2], magnetic resonance electrical impedance tomography (MREIT) ${ }^{[3,4]}$,magnetic induction tomography (MIT) ${ }^{[5,6]}$ and so on.

However, the resolution of these imaging methods is not high. In order to improve resolution, researchers have proposed magneticacoustic imaging technology (MAT) ${ }^{[7]}$. MAT combines the good contrast of electrical impedance tomography with the good spatial resolution of ultrasound imaging. The proposition of MAT has aroused widespread concern in academic circles at home and abroad immediately. Several well-known Research institutions including Department of Biomedical Engineering, University of Minnesota, Chinese Academy of Medical Sciences, Institute of Biomedical Engineering, Zhejiang University, and Institute of Electrical Engineering, Chinese Academy of Sciences. MAT includes Magnetoacoustic Tomography with Magnetic Induction (MAT-MI) ${ }^{[8]}$ and Magnetoacoustic Tomography with Injecting Current (MAT-IC).MAI-MI was proposed by Bin He who is the professor of Department of Biomedical Engineering, University of Minnesota in 2005 year. In MAT-MI a sample

\author{
Guoqiang Liu, Hui Xia \\ Institute of electrical engineering, Chinese \\ Academy of Sciences \\ Beijing, China \\ Email: gqliu@mail.iee.ac.cn; xiahui@mail.iee.ac.cn
}

is placed in a static magnetic field, an excitation coil adds pulsed magnetic stimulation to the sample, and then the eddy current is produced in the samples. The eddy current will generate the Lorentz force with the effect of the static magnetic field. The sample will vibrate and stimulate the ultrasonic signals; through the ultrasonic signals obtained by transducers around the sample we can reconstruct the conductivity distribution of the sample. Magnetoacoustic tomography with injecting current is same in the fundamentals with MAT-MI; the difference is that the alternating current in sample is injected into directly in the former.

In the imaging process, conductivity image of the sample is reconstructed through measuring the ultrasonic signals around the sample; therefore, the accuracy and intensity of ultrasound signals have great influence on the experimental results. In experiment, both static magnetic and electric fields also affect the strength of ultrasonic signal. As the magnetic field is constant, so the intensity of ultrasound signals is determined only by the electrical signals injected into the sample. Therefore, it is very important how to choose the parameters of the electrical signals injected into the sample.

Through experiments we studied the relationship between the Parameters of current signals injected into the sample and the intensity of ultrasound signals collected around the sample. The experiment provides theoretical basis for the selection of power supply used in the subsequent experiments.

\section{THEORY ANALYSIS}

In the experiments of magnetoacoustic tomography with injecting current, a sample is placed in a static magnetic field $\mathbf{B}_{0}$; the alternating current $\mathbf{J}(t)$ is injected into the sample. Due to the interaction between the static magnetic field and the current, acoustic wave can be generated by the Lorentz force that induces acoustic vibration within the sample. The generated acoustic pressure $p$ satisfies the wave equation $(1)^{[9,10]}$

$$
\begin{gathered}
\nabla^{2} p-\frac{1}{c^{2}} \frac{\partial^{2} p}{\partial t^{2}}=\nabla \cdot \mathbf{F} \\
\mathbf{F}=\mathbf{J}(t) \times \mathbf{B}_{0}
\end{gathered}
$$


Where the $F$ is acoustic source inside the sample, $\nabla \cdot$ is the divergence operator, $\nabla^{2}$ is the Laplacian operator and $C$ is the acoustic velocity.

The equation(3)shows the relation between the conductivity and the Lorentz force, the moving charges in a static magnetic field generate the Lorentz force, then the Lorentz force stimulates ultrasonic signal. So we can get more information of the electrical characteristics in the sample by the inverse problem of the electromagnetic field, thus the image reconstruction of conductivity distribution is achieved.

\section{SCHEME and RESULT}

\section{A. Experimental Scheme and Device}

The device used in the experiment are shown in Figure1, a copper rings is the sample in the magnetoacoustic tomography with injecting current, the diameter (D) of the copper ring is $3 \mathrm{~cm}$, and the diameter (d) of the copper line is $1 \mathrm{~mm}$, the frequency of pulse signal provided by the signal generator is adjustable, the center frequency of ultrasonic transducer is $1 \mathrm{MHz}$.

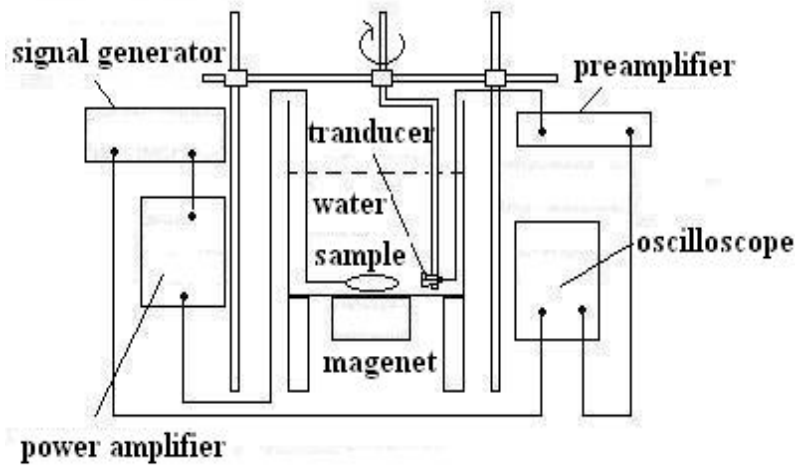

Fig. 1 Experimental device

As is shown in the Figure 1, the copper ring is placed in the container levelly, at the same time the copper ring is in the static magnetic field which direction is vertical, ultrasonic signals generated by copper ring are received by ultrasonic transducer. In order to couple better, copper ring and the ultrasonic transducer are placed in water. The ultrasonic signals received by ultrasonic transducer are transferred into the oscilloscope through the preamplifier. In the oscilloscope we can obtain the information of ultrasound intensity. Ultrasonic signal contains the electrical characteristic information of objects which is measured. Through the reconstruction procedure we can get tissue electrical characteristics.

\section{B. Results and Analysis.}

\section{1) Acquisition of Ultrasonic Signal}

In experiments, square-wave voltage signals are injected into the copper ring, the parameter of the voltage is as follows: amplitude is $5 \mathrm{~V}$, frequency is $2 \mathrm{KHz}$, and duty is
$0.5 \%$. Figure 2 shows the waveforms of ultrasonic signals which are averaged 1024 times by oscilloscope.

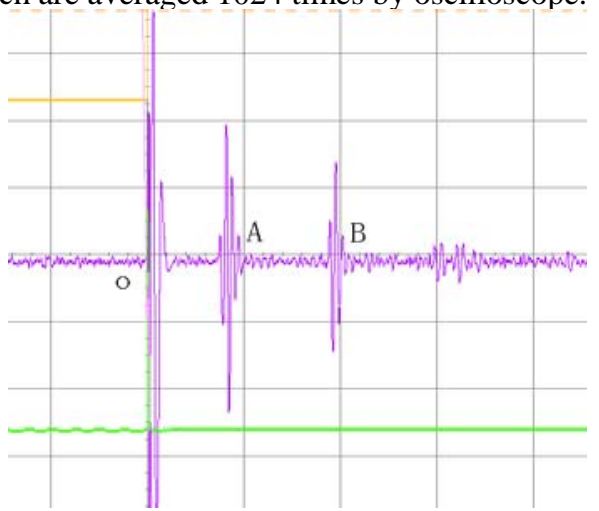

Fig.2 Waveforms of ultrasonic signals

As is shown in Figure 2, there is a delay time of 20us between the two main peaks $\mathrm{A}$ and $\mathrm{B}$. This time interval matches with the distance between the two boundaries of the copper ring. That is the two main peaks corresponding to the two boundaries of copper ring. When moving the ultrasound transducer, the delay time of two peaks is changed, the signals look similarly, but the delay time between the two peaks is constant.

\section{2) Analysis of Current in Cooper Ring}

Ultrasonic signals are produced by electric charge moving in magnetic field, the strength of ultrasonic signals depends on the velocity of the electric charge. The velocity is determined by the magnetic field and current. When the strength of magnetic field is constant, the intensity of ultrasonic signals depend on the current only, accordingly the strength of ultrasonic signals relies on the current only.

In order to get the information of current in the copper ring, a resistor is placed in the circuit; the resistor and the copper ring are in series. So the ratio of the voltage signal and the resistance is the current signal value in the circuit. The circuit of measurement is shown in Figure3.

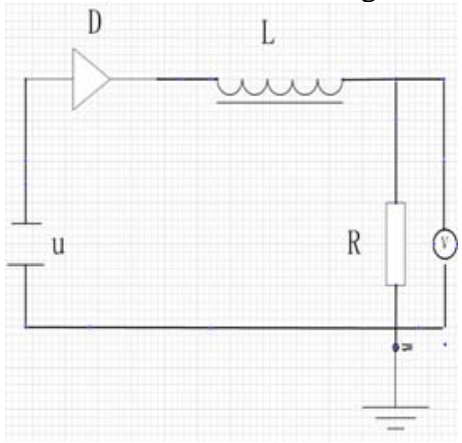

Fig.3 Circuit of measurement

In Figure 3, $\mathrm{L}$ is the copper ring in the experiment, a diode (D) is placed in the circuit to close the oscillation circuit current; $\mathrm{u}$ is the alternating electrical signal injected into the copper ring. $\mathrm{R}$ is the resistance in the circuit. When the Parameter of the voltage is as follows: amplitude is $5 \mathrm{~V}$, frequency is $1.2 \mathrm{KHz}$, and duty is $0.5 \%$, the waveform of current in the copper ring is shown in Figure4. 


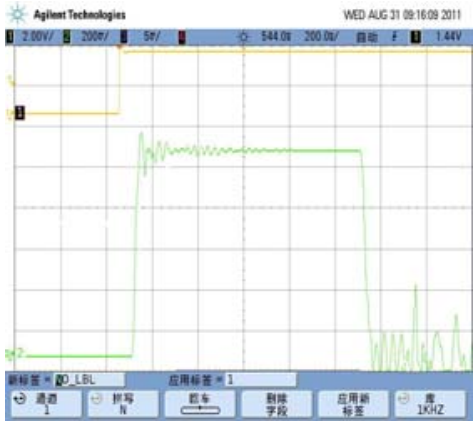

Fig.4 Waveforms of current

When the frequency of the current is $1.2 \mathrm{KHz}$, the pulse width of current is $1000 \mathrm{~ns}$; as the frequency becomes smaller, the current pulse width becomes larger. In the experiments, we change the frequency of square wave signals to obtain the relation between the ultrasound signal and the current, the relation of ultrasound signal and the current frequency is as shown in Figure5.

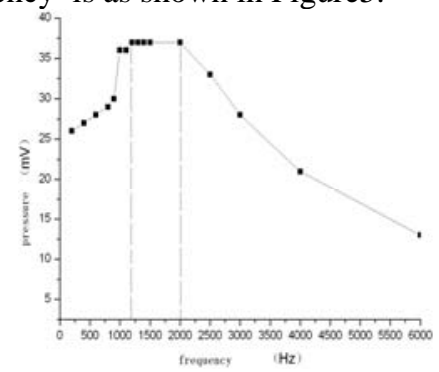

Fig.5 Relations between the ultrasonic signal and frequency

We can see from Figure5, the strength of ultrasonic signals is strongest when the frequency of current change is from $1.2 \mathrm{KHz}$ to $2 \mathrm{KHz}$; the biggest value of strength is $37 \mathrm{mv}$ or so. From the former experiment we know that the pulse width of current becomes from 500ns to 1000ns when the frequency of current change is from $1.2 \mathrm{KHz}$ to $2 \mathrm{KHz}$. So we can get the conclusion that the strength of ultrasonic signals is strong when the pulse width of current becomes from $500 \mathrm{~ns}$ to $1000 \mathrm{~ns}$.

3) Imaging Experiments of Copper Ring

Using the results of experiments above, we design an experiment to reconstruct the divergence of Lorenz force. The pulse width of current injected into the copper ring is 600 ns.Schematic diagram of signal acquisition is shown in Fig.6.

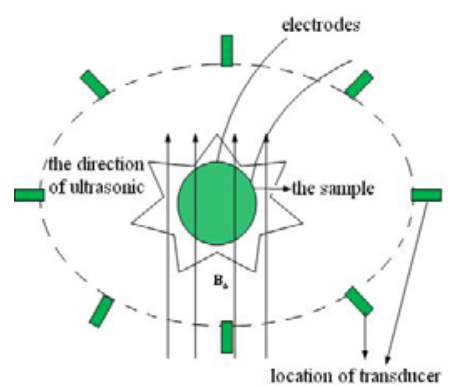

Fig.6 Schematic diagram of signal acquisition

The position of transducer is as Figure6, the distance between the copper ring and the transducer is $6 \mathrm{~cm}$. We got a set of ultrasonic signals by rotating the transducer. Figure7 shows an example of reconstructed the divergence of Lorenz force using the MAT-IC approach described above.

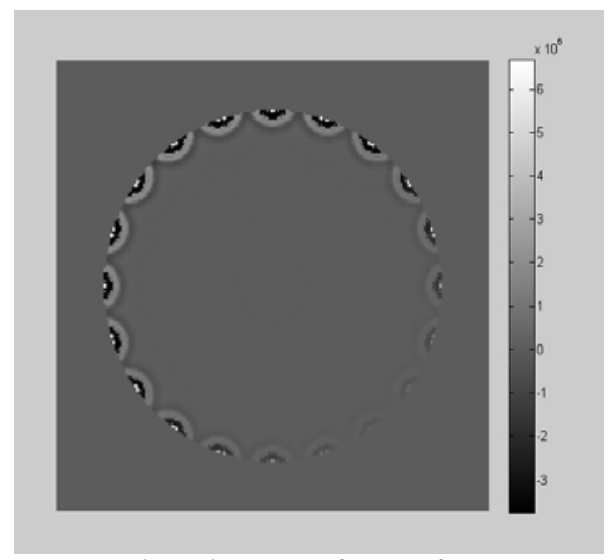

Fig.7 Divergence of Lorenz force

As in Figure.7, the 2D MAT-IC image agrees well with the shape and size of the conductivity boundary of copper ring.

\section{ACKNOWLEDGMENT}

In this experiment, the selection of pulse source is very important; frequency of power influences the intensity of ultrasonic signal directly. Therefore, we made lots of works on the parameters of power and got experiences on the selection of power. The conclusion of experiments shows that: when the pulse width of current in the ring ranges from 500ns to $1000 \mathrm{~ns}$, ultrasonic signal stimulated by Lorenz force is the strongest. Therefore, as long as the pulse width of current change from 500ns to 100ns, we can obtain strong ultrasonic signal. This conclusion provides theoretical basis for the selection of power supply used in the subsequent experiments.

This work is supported by the National Natural Science Foundation of China (No.50977084, 60802086, 61002036, and 51137004) and Beijing Nova Program-2009B48

\section{REFERENCES}

[1] J. G. Webster, "Electrical Impedance Tomography,” Adam Hilger, Bristol, UK, 1990.

[2] P. Metheral, D. C. Barber, R. H. Smallwood, and B. H. Brown, "Three-dimensional electrical impedance tomography," Nature, vol. 380, pp. 509-512, 1996.

[3] O. Kwon, E. Woo, J. Yoon, and J. K. Seo, "Magnetic resonanceelectrical impedance tomography (MREIT) Simulation study of Jsubstitutionalgorithm,” IEEE Trans. Biomed. Eng, vol. 49, pp. 160-167, 2002.

[4] N. Gao, S. Zhu, and B. He, "Estimation of electrical conductivity distribution within the human head from magnetic flux density measurement," Physics in Medicine and Biology, vol. 50, pp. 26752687, 2005.

[5] A. J. Peyton, Z. Z. Yu, G. Lyon, S. Al-Zeibak, J. Ferreira, J. Velez, F. Linhares, A. R. Borges, H. L. Xiong, N. H. Saunders, and M. S. Beck, "An overview of electromagnetic inductance tomography:description of three different systems," Measurement Science and Technology, vol. 7, pp. 261-271, 1996.

[6] H. Griffiths, "Magnetic Induction tomography," Measurement science and technology, vol. 12, pp. 1126-1131, 2001. 
[7] B. C. Towe and M.R. Islam, "A magneto-acoustic method forthe noninvasive measurement of bioelectric currents," IEEE Trans. Biomed.Eng., vol. 35, pp. 892-894, 1988.

[8] Y. Xu and B. He, "Magnetoacoustic tomography with magnetic induction (MAT-MI)," Physics in Medicine and Biology, vol. 50, pp. 5175-5187, 2005.

[9] P. M. Morse and H. Feshbach, "Methods of Theoretical Physics," New York, 1953.

[10] G. H. Du, Z. M. Zhu, and X. F. Gong, "Fundamentals of Acoustics," Nanjing: Nanjing University Press, 2001.

[11] X. Li, "A method of bio-tissue imaging coupling magnetic,acoustic and electric fields," Zhejiang University, pp. 17-19, 2006. 